\title{
Association between painful temporomandibular disorders, sleep bruxism and tinnitus
}

Giovana FERNANDES(a)

José Tadeu Tesseroli de SIQUEIRA(b)

Daniela Aparecida de Godoi GONÇALVES(a)

Cinara Maria CAMPARIS(a)

(a) Department of Dental Materials and Prosthodontics, Araraquara School of Dentistry, Univ Estadual Paulista - UNESP, Araraquara, SP, Brazil.

(b) Department of Neurology, Hospital das Clínicas, Universidade de São Paulo - USP, São Paulo, SP, Brazil.

Declaration of Interests: The authors certify that they have no commercial or associative interest that represents a conflict of interest in connection with the manuscript.

Corresponding Author:

Giovana Fernandes

E-mail: giovana_fernandes@hotmail.com

http://dx.doi.org/10.1590/1807-3107BOR-2014.vol28.0003

Submitted: April 4, 2013

Accepted for publication: Nov 13, 2013

Last revision: Jan 6, 2014
Abstract: The present cross-sectional study was designed to investigate the association between sleep bruxism (SB), tinnitus and temporomandibular disorders (TMD). The sample consisted of 261 women (mean age of 37.0 years). The Research Diagnostic Criteria for Temporomandibular Disorders were used to classify TMD and self-reported tinnitus. SB was diagnosed by clinical criteria proposed by the American Academy of Sleep Medicine. The results showed an association between painful TMD and tinnitus ( $\mathrm{OR}=7.3 ; 95 \% \mathrm{CI}=3.50-15.39 ; \mathrm{p}<0.001)$. With regard to $\mathrm{SB}$, the association was of lower magnitude $(\mathrm{OR}=1.9 ; 95 \% \mathrm{CI}=1.16-3.26$; $\mathrm{p}<0.0163$ ). When the sample was stratified by the presence of $\mathrm{SB}$ and painful TMD, only SB showed no association with tinnitus. The presence of painful TMD without SB was significantly associated with tinnitus $(\mathrm{OR}=6.7 ; 95 \% \mathrm{CI}=2.64-17.22 ; \mathrm{p}<0.0001)$. The concomitant presence of painful TMD and SB was associated with a higher degree of tinnitus severity $(\mathrm{OR}=7.0 ; 95 \% \mathrm{CI}=3.00-15.89 ; \mathrm{p}<0.0001)$. It may be concluded that there is an association between SB, painful TMD and self-reported tinnitus; however, no relationship of a causal nature could be established.

Keywords: Facial Pain; Sleep Bruxism; Tinnitus.

\section{Introduction}

Temporomandibular disorder (TMD) is a collective term that embraces a number of clinical problems involving the masticatory muscle, temporomandibular joints (TMJs), and associated structures. ${ }^{1}$ The etiology of TMD has been considered multifactorial, because one or more factors may contribute to its predisposition, initiation, and maintenance. Among these factors, sleep bruxism (SB) may be involved in triggering and/or maintaining TMD. ${ }^{1}$ The American Academy of Sleep Medicine (AASM) classified SB as a stereotyped movement disorder occurring during sleep and characterized by tooth grinding and/or clenching. ${ }^{2}$

Patients with TMD often report tinnitus as an associated symptom, and the relationship between the two is still only partially understood.,4 Tinnitus can be defined as the perception of sound or noise in the absence of an evident external stimulus. ${ }^{5}$ Approximately $10 \%$ to $15 \%$ of the general population complains of tinnitus, and its prevalence increases with age. However, the prevalence of tinnitus in TMD patients ranges from $33 \%$ to $76 \%$, which is a much higher rate than that of the general population. . $^{6,8,9,10}$ 
Considering the prevalence of both SB and tinnitus in TMD patients, it has been noted that there are only few studies on this topic. A high prevalence of tinnitus has been found in patients with $\mathrm{SB}$ and $\mathrm{TMD}_{,}^{11}$ but it is not yet known how SB, TMD and tinnitus are associated, or how one influences the other. Based on these considerations, the aim of the present study was to investigate a possible triple association between these three factors (painful TMD, SB, and tinnitus), and assess the odds of tinnitus in patients with or without painful TMD and SB.

\section{Methodology} Study Design

This investigation was designed as a cross-sectional study. The sample consisted of 261 women recruited consecutively among patients with the chief complaint of orofacial pain, who sought care at a university-based specialty clinic (Universidade Estadual Paulista, Araraquara, SP).

The exclusion criteria were: odontalgia (toothache), neuropathy, intraoral lesions, any chronic pain syndrome (including fibromyalgia or arthritis), cognition or language impairments and individuals under the 18 years of age. All patients included in the study presented stability of vertical dimension with posterior dental support provided by natural teeth or partial removable or fixed dental prosthesis.

This study was approved by the Local Research Ethics Committee (Protocol no. 196/96). All patients read, understood, and signed a Term of Free and Informed Consent.

\section{Assessment}

All individuals were evaluated by one single calibrated researcher according to standardized methods of assessment, as noted below:

a. Orofacial Pain Clinic Protocol: all participants were interviewed and underwent systematic examination of cervical, cranial, facial, dental, and other oral structures. The objectives were to determine: the chief complaint, TMD pain characteristics (location, intensity, quality, duration, exacerbating factors), and medical history. The American Academy of Orofacial Pain diagnos- tic criteria were applied in performing the differential diagnoses in relation to other conditions that may mimic TMD. In the cases with confirmed TMD diagnosis, the classification was made according to the RDC/TMD criteria.

b. Research Diagnostic Criteria for Temporomandibular Disorders (RDC/TMD): the RDC/ $\mathrm{TMD}^{12}$ is a standardized system for diagnosing and classifying the most common subtypes of TMD, by means of a questionnaire and a physical examination. In this study a Portuguese version was used. ${ }^{13,14,15}$ Based on Axis I of the RDC/TMD, the patients were stratified according to their TMD status, as follows: Group 1: Patients with painful TMD, according to Group I of the RDC/TMC (myofascial pain TMD, or myofascial pain TMD with limited opening) and/or Group III of the RDC/TMC (TMJ arthralgia and/or osteoarthritis), and Group 2: Patients without painful TMD, according to Group II of the RDC/TMD (disc displacement with or without reduction), or else diagnosed with no TMD. All patients presented chronic pain according to the International Association for the Study of Pain criteria. ${ }^{16}$ The diagnosis of tinnitus was obtained by self-report of ear noises in answer to the question, "Do you have noises or ringing in your ears?" on the RDC/TMD questionnaire.

c. Clinical diagnostic criteria for Sleep Bruxism was met when the patient reported or was aware of the sounds of tooth grinding during sleep and at least one of the following adjunctive criteria: a) observation of abnormal tooth wear; $b$ ) report of morning masticatory muscle fatigue or pain; and c) masseteric hypertrophy upon digital palpation. In addition, there was no better explanation for the jaw muscle activity afforded by any other current sleep disorder, medical or neurological disorder, medication use or substance use disorder. These criteria were in accordance with the clinical diagnostic criteria proposed by AASM. ${ }^{2}$

\section{Data analysis}

Descriptive statistics and frequency counts were used to characterize the sample. The sample was 
Table 1. Demographic characteristics of the sample.

\begin{tabular}{|c|c|c|}
\hline Total Sample & $\mathrm{N}$ & $\%$ \\
\hline \multicolumn{3}{|l|}{ Gender } \\
\hline Women & 261 & 100.0 \\
\hline \multicolumn{3}{|l|}{ Age } \\
\hline Mean & 37.0 & \\
\hline SD & 12.7 & \\
\hline Range & $18-76$ & \\
\hline \multicolumn{3}{|l|}{ Educational level } \\
\hline$\leq \_$Middle School & 62 & 23.8 \\
\hline High School & 107 & 41.0 \\
\hline College & 81 & 31.0 \\
\hline Missing & 11 & 4.2 \\
\hline \multicolumn{3}{|l|}{ Marital status } \\
\hline Married & 150 & 57.5 \\
\hline Single & 75 & 28.7 \\
\hline Separated/divorced & 26 & 10.0 \\
\hline Widower & 9 & 3.4 \\
\hline Missing & 1 & 0.4 \\
\hline \multicolumn{3}{|l|}{ Race } \\
\hline Caucasian & 204 & 78.2 \\
\hline Black & 23 & 8.8 \\
\hline Brown & 18 & 6.9 \\
\hline Asiatic & 1 & 0.4 \\
\hline Missing & 15 & 5.7 \\
\hline
\end{tabular}

stratified according to self-reported tinnitus to study the association with painful TMD and with the presence of SB, and also to investigate the presence of painful TMD among SB patients. Furthermore, the sample was stratified according to the absence or presence of painful TMD and SB.

Statistical analyses were conducted using SPSS 15.0 for Windows (SPSS Inc., Chicago, USA). The Chisquare $\left(\chi^{2}\right)$ test and odds ratio (OR) with a $95 \%$ confidence interval (CI) were applied, and the significane level adopted was 0.05 . The nonparametric MannWhitney test was used for quantitative variables.

\section{Results}

Two hundred and sixty one women were evaluated. The mean age was 37.0 years, with ages ranging from 18 to 76 years. The majority of the individuals had completed high school $(41.0 \%)$, were married (57.5\%), and were Caucasian (78.2\%) (Table 1).

The medical history of tinnitus patients reported the following diseases: $28.2 \%$ gastritis/ulcer $(p=0.808)$, $8.7 \%$ arterial hypertension $(\mathrm{p}=0.073), 2.0 \%$ hepatitis $(p=0.623), 41.6 \%$ sinusitis/rhinitis $(p=0.227), 5.4 \%$ heart disease $(p=0.205), 4.0 \%$ diabetes $(p=0.482)$, $34.2 \%$ spine alterations $(p=0.118)$ and $6.0 \%$ asthma/ bronchitis $(p=0.378)$. None of the patients reported hearing loss or medication abuse. The use of medications was recorded, but the variety of medications stated precluded frequencies or group comparisons from being determined.

The present study showed that painful TMD was associated with the presence of tinnitus ( $p<0.0001)$, since $93.3 \%$ of the individuals presenting tinnitus also presented painful TMD, versus $6.7 \%$ without painful TMD (OR = 7.3; 95\%CI = 3.50-15.39) (Table 2). When considering age, the nonparametric Mann-Whitney test showed no significant difference between groups with and without self-reported tinnitus $(\mathrm{p}=0.554)$. Association was also observed between SB and the presence of self-reported tinnitus ( $\mathrm{p}=0.0163)$, but to a lower degree $(\mathrm{OR}=1.9 ; 95 \% \mathrm{CI}=1.16-3.26)$ (Table 3$)$.

Considering only SB patients, a higher magnitude of association was found between painful TMD and selfreported tinnitus $(\mathrm{OR}=7.1 ; 95 \% \mathrm{CI}=1.40-36.88)$ (Table 4). When the sample was stratified according to the absence or presence of SB and painful TMD (Table5), association with self-reported tinnitus was found to be present for painful TMD without $\mathrm{SB}(\mathrm{OR}=6.7 ; 95 \% \mathrm{CI}=2.64-17.22)$. The concomitant presence of painful TMD and SB significantly increased the magnitude of association with self-reported tinnitus ( $\mathrm{OR}=7.0 ; 95 \% \mathrm{CI}=3.00-15.89)$.

Table 2. Association between painful TMD and self-reported tinnitus.

\begin{tabular}{|c|c|c|c|c|}
\hline & \multicolumn{2}{|c|}{ Painful TMD } & \multirow{2}{*}{ Total } & \multirow{2}{*}{ OR $(95 \%$ IC) } \\
\hline & Absence & Presence & & \\
\hline Without Tinnitus n (\%) & $34(34.7)$ & $64(65.3)$ & $98(100.0)$ & Reference \\
\hline With Tinnitus n (\%) & $11(6.7)$ & $152(93.3)$ & 163 (100.0) & $7.3(3.50-15.39)$ \\
\hline Total n (\%) & 45 (17.2) & $216(82.8)$ & 216 (100.0) & $p<0.0001$ \\
\hline
\end{tabular}


Table 3. Association between sleep bruxism and self-reported tinnitus.

\begin{tabular}{|c|c|c|c|c|}
\hline & \multicolumn{2}{|c|}{ Sleep Bruxism } & \multirow{2}{*}{ Total } & \multirow{2}{*}{ OR $(95 \% \mathrm{Cl})$} \\
\hline & Absence & Presence & & \\
\hline Without Tinnitus n (\%) & $46(46.9)$ & $52(53.1)$ & $98(100.0)$ & Reference \\
\hline With Tinnitus n (\%) & $51(31.3)$ & $112(68.7)$ & $163(100.0)$ & $\begin{array}{c}1.9(1.16-3.26) \\
p=0.0163\end{array}$ \\
\hline Total n (\%) & $97(37.2)$ & $164(62.8)$ & $261(100.0)$ & \\
\hline
\end{tabular}

\section{Discussion}

The present study investigated the concomitant presence of painful TMD, SB and tinnitus. Since all these conditions are highly prevalent and have a great impact on an individual's life, we feel that our findings can contribute to the currently available knowledge. The most important findings were: (1) There was an association between painful TMD and self-reported tinnitus; (2) the same was found for SB, but at a lower magnitude; (3) association with selfreported tinnitus was found in patients with painful TMD and without SB and also in patients with painful TMD and with SB and, but at a higher magnitude.

The present study demonstrated the association between TMD pain and tinnitus. Recent studies have also demonstrated this association; TMD pain seems to be a risk factor for the occurrence of tinnitus. ${ }^{4,6,78,9,10}$ TMD covers many types of diagnoses classified according the RDC/TMD. In the present study, the painful TMD group included a majority of patients with arthralgia and myofascial pain combined. Only nine (9) patients had arthralgia not combined with myofascial pain, as assessed by the RDC/TMD; this precluded analysis of the association between tinnitus and each of these diagnoses (arthralgia and myofascial pain). However, these points reflect an important characteristic of the sample: it is very rare to find individuals presenting chronic TMD with only myofascial pain or arthralgia.
The most plausible hypothesis for the pain-tinnitus relationship is that it may require two components. ${ }^{17}$ First, auditory connections with the trigeminal system occur at the very lowest levels of each sensory system, where small cells in the trigeminal ganglia send excitatory unmyelinated or lightly myelinated axons to terminate in the cochlear nucleus $(\mathrm{CN}){ }^{18,19}$ Based on this interaction, the gracile and cuneate nuclei present in the somatosensory pathway, once sensitized, would engender painful stimuli generated from the somatosensory pathway to the auditory cortex, which, in turn, interprets these stimuli as tinnitus. 17,18,19,20,21 Second, there are descending modulatory pathways - a modulation system that contributes to pain relief - in the central processing of pain. Clinical evidence supports the emerging view that this system may play an important role in maintaining chronic pain. ${ }^{22}$ Continuous somatosensory impulses from painful TMD could lead to dysfunction of the descending modulatory pathways, resulting in an increase in neural somatosensory system impulses to the cochlear nuclei, leading, in turn, to consolidation of plastic changes in the auditory system, thus contributing to tinnitus chronicity. ${ }^{17}$

There is no hypothesis in the literature about a specific association between SB and tinnitus. Only one study showed a high prevalence of tinnitus in patients with SB and painful TMD. ${ }^{11}$ The present study showed that $68.7 \%$ of SB patients had tinnitus,

Table 4. Association between sleep bruxism and painful TMD diagnosed according self-reported tinnitus.

\begin{tabular}{lccccc} 
& \multicolumn{2}{c}{ Sleep Bruxism } & & \multirow{2}{*}{ Total } & OR (95\%Cl) \\
\cline { 2 - 3 } & No Painful TMD & Painful TMD & & & \\
\hline Without Tinnitus n (\%) & $6(11.5)$ & $46(88.5)$ & & $52(100.0)$ & Reference \\
With Tinnitus n (\%) & $2(1.8)$ & $110(98.2)$ & & $112(100.0)$ & $7.1(1.40-36.88)$ \\
& & & & 0.0210 & $p=0$ \\
\hline Total n (\%) & $8(4.9)$ & $156(95.1)$ & & $164(100.0)$ & \\
\hline
\end{tabular}


Table 5. Association between self-reported tinnitus, temporomandibular disorders and sleep bruxism.

\begin{tabular}{|c|c|c|c|c|}
\hline & \multicolumn{3}{|c|}{ Tinnitus } & \multirow{2}{*}{ OR (95\%CI) } \\
\hline & Absence n (\%) & Presence n (\%) & Total n (\%) & \\
\hline - painful TMD - SB & $26(26.5)$ & $9(5.5)$ & $35(13.4)$ & Reference \\
\hline - painful TMD + SB & $8(8.2)$ & $2(1.2)$ & $10(3.8)$ & $\begin{array}{c}0.7(0.13-4.06) \\
p=0.7108\end{array}$ \\
\hline+ painful TMD - SB & $18(18.4)$ & $42(25.8)$ & $60(23.0)$ & $\begin{array}{c}6.7(2.64-17.22) \\
p<0.0001\end{array}$ \\
\hline+ painful TMD + SB & $46(46.9)$ & $110(67.5)$ & $156(59.8)$ & $\begin{array}{c}7.0(3.00-15.89) \\
p<0.0001\end{array}$ \\
\hline Total & $98(100.0)$ & 163 (100.0) & $261(100.0)$ & \\
\hline
\end{tabular}

implying that this magnitude of association between $\mathrm{SB}$ and tinnitus is unprecedented in the literature.

Therefore, with regard to SB, it could not be directly associated with tinnitus, but it could be associated with painful TMD. The association between SB and painful TMD has been demonstrated in clinical studies. ${ }^{23,24,25}$ These studies have shown that $58 \%$ to $80 \%$ of TMD individuals present SB. ${ }^{23,24}$ Furthermore, SB has been found more frequently in patients with painful TMD conditions, such as myofascial pain and arthralgia. ${ }^{25}$ The pain experienced by patients with SB may be analogous to mechanical, overloadinduced post-exercise muscle soreness. ${ }^{26,27}$ It is characterized by pain and inflammation that develop gradually over several hours and then gradually diminish over a few days. ${ }^{27}$ This source of ongoing pain could trigger and perpetuate TMD. ${ }^{27}$ Thus, our results showed that patients with painful TMD presented a greater risk for acquiring tinnitus, and that this risk was greater in the presence of SB.

Our study had certain limitations. It was a crosssectional study, and, as such, no cause-and-effect relationship could be made among sleep bruxism, tinnitus and TMD. The sample consisted of patients who sought treatment for TMD, and the results are not representative of the general population. This high prevalence of tinnitus was not found in an overall population sample. ${ }^{5}$ In addition, the presence of tinnitus was verified by the patients' self-reports. The RDC/TMD questionnaire has other questions in regard to the self-reporting of TMJ clicking and crepitus, respectively: "Does your jaw click or pop when you open or close your mouth or when chewing?" and "Does your jaw make a grating or grinding noise when it opens and closes or when chewing?".
It could be that, in our study, the patients mistook the question about tinnitus (Do you hear noises or ringing in your ears?) for those about TMJ clicking or crepitus. These questions are actually very different, insofar as the sound for TMJ clicking and crepitus is related to a mandibular function. Moreover, we found only $27(16.8 \%)$ patients had tinnitus self-reports and TMJ clicking, and $20(12.4 \%)$ patients had tinnitus and crepitus, both verified by stethoscope auscultation during the RDC/TMD physical examination. The diagnosis of SB has previously been discussed, and polysomnographic records have been considered a more precise diagnosis of $\mathrm{SB} ;{ }^{28}$ however, for large samples, polysomnographic technology is still expensive and inaccessible. In regard to the clinical aspects, however, the best way of establishing a uniform and more accurate sample of these patients was to obtain reports of tooth grinding confirmed by a roommate or a family member, and obtain the others signs and symptoms according to observations made from the clinical criteria proposed by the AAMS. ${ }^{2}$ In this case, the patients who meet these criteria probably have SB. ${ }^{28}$ Moreover, the presence of diseases was verified by the patients' self reports, without the involvement of an otolaryngologist or other medical specialist; that is to say, patients reported the diseases which they knew about.

On the other hand, our methodology had important strengths: TMD was diagnosed and classified by RDC/TMD, in accordance with the gold standard. The assessments were made by a trained researcher, thus improving the reliability of the data. Moreover, to our knowledge it was the first study that simultaneously investigated the associations between painful TMD, SB and tinnitus. 


\section{Conclusion}

In conclusion, the present study shows associations between SB, painful TMD and tinnitus. Sleep bruxism patients presented increased odds of acquiring painful TMD. Furthermore, patients with painful TMD presented an increased risk of acquiring tinnitus and this risk was greater in the presence of SB. Hence, patients with sleep bruxism and painful TMD may present more severe and complex clinical findings, such as tinnitus observed through selfreport. In these cases, it is essential that the association between tinnitus and other systemic diseases be investigated in clinical practice, and that these patients be referred for further medical evaluation. As regards the management of painful TMD,

\section{References}

1. McNeill C. Management of temporomandibular disorders: concepts and controversies. J Prosthet Dent. 1997 May;77(5):510-22.

2. Kato T, Thie NMR, Montplasir JY, Lavigne GJ. Bruxism and orofacial movements during sleep. Dent Clin North Am. 2001 Oct;45(4):657-684.

3. Hilgenberg PB, Saldanha AD, Cunha CO, Rubo GH, Conti PC. Temporomandibular disorders, otologic symptoms and depression levels in tinnitus patients. J Oral Rehabil. 2012 Apr;39(4):239-44.

4. Bernhardt O, Mundt T, Welk A, Köppl N, Kocher T, Meyer $\mathrm{G}$, et al. Signs and symptoms of temporomandibular disorders and the incidence of tinnitus. J Oral Rehabil. 2011 Dec;38(12):891-901.

5. Ahmad N, Seidman M. Tinnitus in the older adult: epidemiology, pathophysiology and treatment options. Drugs Aging. 2004;21(5):279-305.

6. Chole RA, Parker WS. Tinnitus and vertigo in patient with temporomandibular disorder. Arch Otolaryngol Head Neck Surg. 1992 Aug;118(8):817-21.

7. Gelb H, Gelb ML, Wagner ML. The relationship of tinnitus to craniocervical mandibular disorders. Cranio. 1997 Apr;15(2):136-43.

8. Lam DK, Lawrence HP, Tenenbaum HC. Aural symptoms in temporomandibular disorder patient attending a craniofacial pain unit. J Orofac Pain. 2001 Spring;15(2):146-57.

9. Tuz HH, Onder EM, Kisnisci RS. Prevalence of otologic complaints in patients with temporomandibular disorder. Am J Orthod Dentofacial Orthop. 2003 Jun;123(6):620-3.

10. Bernhardt O, Gesch D, Schwahn C, Bitter K, Mundt T, Mack $\mathrm{F}$, et al. Signs of temporomandibular disorders in tinnitus our recommendation to clinical professionals is to control the risk factors of TMD maintenance and aggravation, such as sleep bruxism. When no reasonable cause for tinnitus is found, such as a local or systemic condition, we now know that, unfortunately, although controlling pain and central sensitization cannot eliminate tinnitus, sometimes controlling pain can help a patient assuage tinnitusrelated discomfort. ${ }^{29}$

\section{Acknowledgements}

The authors would like to thank the Coordenação de Aperfeiçoamento de Pessoal de Nivel Superior (Capes) for their financial support, and the research participants for their invaluable contribution to this study.

patients and in a population-based group of volunteers: results of the study of health in Pomerania. J Oral Rehabil. 2004 Apr;31(4):311-19.

11. Camparis CM, Formigoni G, Teixeira MJ, Siqueira JTT. Clinical evaluation of tinnitus in patients with sleep bruxism: prevalence and characteristics. J Oral Rehabil. 2005 Nov;32(11):808-14.

12. Dworkin SF, LeResche L. Research diagnostic criteria for temporomandibular disorders: review, criteria, examinations and specifications, critique. J Craniomand Disord. 1992 Fall;6(4):301-55.

13. Pereira Júnior FJ, Favilla EE, Dworkin S, Huggins K. Critérios de diagnóstico para pesquisa das disfunções temporomandibulares (RDC/TMD). Tradução oficial para a língua portuguesa. J Bras Clin Odontol Integr. 2004 Out-Dec;8(47):384-95.

14. Kosminsky M, Lucena LBS, Siqueira JTT, Pereira Júnior F, Góes PSA. Adaptação cultural do questionário Research Diagnostic Criteria for Temporomandibular Disorders: Axis II para o português. J Bras Clin Odontol Int. 2004 JanFev;8(43):51-61.

15. Lucena LBS, Kosminsky M, Costa LJ, Góes PSA. Validation of the Portuguese version of the RDC/TMD Axis II questionnaire. Braz Oral Res. 2006 Out-Dec;20(4):312-7.

16. Merskey NB. Classification of chronic pain: descriptions of chronic pain syndromes and definitions of pain terms prepared by the International Association for the Study of Pain. Seatlle: IASP Press; 1994.

17. Salvinelli F, Casale M, Paparo F, Persico AM, Zini C. Subjective tinnitus, temporomandibular joint dysfunction, and serotonin modulation of neural plasticity: causal or casual triad?. Med Hypotheses. 2003 Oct;61(4):446-8. 
18. Shore S, Zhou J, Koehler S. Neural mechanisms underlying somatic tinnitus. Prog Brain Res. 2007;166:107-23.

19. Shore SE. Plasticity of somatosensory inputs to the cochlear nucleus -implications for tinnitus. Hear Res. 2011 Nov;281(1-2):38-46.

20. Levine RA. Somatic (craniocervical) tinnitus and the dorsal cochlear nucleus hypotheses. Am J Otolaryngol. 1999 NovDec;20(6):351-62.

21. Cacace AT. Expanding the biological basis of tinnitus: crossmodal origins and the role of neuroplasticity. Hear Res. 2003 Jan;175(1-2):112-32.

22. Ossipov MH, Dussor GO, Porreca F. Central modulation of pain. J Clin Invest. 2010 Nov;120(11):3779-87.

23. Camparis CM, Siqueira JTT. Sleep Bruxism: clinical aspects and characteristics in patients with and without chronic Orofacial pain. Oral Surg Oral Med Oral Pathol Oral Radiol Endod. 2006 Feb;101(2):188-93.

24. Huang GJ, LeResche L, Critchlow CW, Martin MD, Drangsholt MT. Risk factors for diagnostic subgroups of painful temporomandibular disorders (TMD). J Dent Res. 2002 Apr;81(4):284-8.

25. Molina OF, Santos Júnior J. The prevalence of some joint disorders in craniomandibular disorder (CMD) and bruxers as compared to CMD nonbruxer patients and controls. Cranio. 1999 Jan;17(1):17-29.

26. Arima T, Svensson P, Arendt-Nielsen L. Experimental grinding in healthy subjects: a model for postexercise jaw muscle soreness. J Orofac Pain. 1999 Spring;13(2):104-14.

27. Lund JP, Lavigne GJ, Dubner R, Sessle BJ. Orofacial pain: from basic science to clinical management. São Paulo: Quintessence; 2002. $260 \mathrm{p}$.

28. Lobbezzo F, Ahlberg J, Glaros AG, Kato T, Koyano K, Lavigne GJ, et al. Bruxism defined and graded: an international consensus. J Oral Rehabil. 2013 Jan;40(1):2-4.

29. Wright EF. Otologic symptom improvement through TMD therapy. Quintessence Int. 2007 Oct;38(9):564-71. 